\title{
Invisible Metallic Microfiber in Clothing Presents Unrecognized MRI Risk for Cutaneous Burn
}

\author{
J.A. Pietryga, M.A. Fonder, J.M. Rogg, D.L. North, and L.G. Bercovitch
}

\section{ABSTRACT}

SUMMARY: We report a case of a thermal burn that occurred during MR imaging likely caused by invisible silver-embedded microfibers in the fabric of an undershirt. As the prevalence of fabric containing nondetectable metallic microfiber increases in athletic and "tech" clothing, the importance of having patients change into safe facility-provided garments before MR imaging is emphasized.

ABBREVIATIONS: $A C R=$ American College of Radiology; $S A R=$ specific absorption rate; $S M F=$ silver microfiber

$\mathbf{R}$ igorous safety screening before MR imaging is essential to prevent injury from non-MR imaging - compatible materials or devices entering the scanner environment. Despite careful screening procedures, accidents have occurred, resulting in serious patient injury, including death. ${ }^{1,2}$ Among the injuries sustained during MR imaging examination are numerous cases of thermal burns. ${ }^{3-5}$ Because of normal MR imaging heating effects and safety constraints, patients are encouraged to change into hospital garments or wear loose-fitting non-metal-containing clothing for their examinations. We report a case of a seconddegree burn sustained by a pediatric patient caused by a typicalappearing gray undershirt later found to contain SMF. This case illustrates the importance of having all patients, especially those undergoing sedation, change into safe, controlled, hospital-provided garments before their MR imaging examination.

\section{TECHNIQUE}

A 71-kg 11-year-old girl presented for outpatient MR imaging of the spine for evaluation of scoliosis, wearing a gray undershirt under a long-sleeved white tee shirt and gray sweat pants. The undershirt was longer than the overshirt and was visible. There were no snaps, zippers, embroidery, or other discernable evidence of metal on or in the patient's clothing. The patient was screened verbally, visually, and with a wand metal detector (SuperWand; Garrett, Garland, Texas) before entering the scanner room. The

Received May 13, 2011; accepted after revision June 27.

From the Departments of Diagnostic Imaging (J.A.P., J.M.R.), Dermatology (M.A.F. L.G.B.), and Medical Physics (D.L.N.), The Warren Alpert Medical School of Brown University/Rhode Island Hospital, Providence, Rhode Island.

Please address correspondence to Jeffrey M. Rogg, MD, Department of Diagnostic Imaging, Rhode Island Hospital, 593 Eddy St, Providence, RI 02903; e-mail: jrogg@ lifespan.org

http://dx.doi.org/10.3174/ajnr.A2827 study was performed by using a wide-bore $1.5 \mathrm{~T}$ imaging system equipped with 33-mT/m gradients (Espree; Siemens, Erlangen, Germany) by using a standard receive-only 8 -element spine-array coil integrated with a 2-element neck-array coil (Siemens). Our routine scanning protocol for scoliosis evaluation was followed (Table). All scanning sequences complied with FDA SAR guidelines. Intravenous propofol sedation was administered during the examination. The patient's arms were fastened to her lateral trunk bilaterally with the volar forearms and palms facing inward by using nylon-Velcro straps (Velcro, Manchester, New Hampshire) to avoid arm-to-magnet-bore-sidewall contact. Careful attention was paid to avoiding skin-to-coil and skin-to-skin contact and contact with conductive cable loops.

Following patient arousal from sedation, she complained of right-sided "burning" discomfort. Physical examination revealed linear erythematous blistering eruptions located along her right flank (Fig 1) and ipsilateral volar wrist where it contacted the undershirt. A dermatologic consult diagnosed a second-degree burn.

Scan sequences, SAR parameters, and coil and cable placements during the examination were reviewed, and no deviation from normal parameters was found. Review of the images showed no evidence of artifacts on the thoracic series, which included the site of blistering, though the specific location of the cutaneous flank burn was not included within the image FOV. While exploring potential sources for the burn with the patient and her family, the patient noted that her undershirt was called the "Boston Silver T" by the National Orthotics and Prosthetics Company store (Boston Brace International, Avon, Massachusetts), where it was purchased (Fig 2). The family had not been informed that the shirt contained SMF. The shirt contents were identified as Coolmax/ Lycra, (INVISTA, Wichita, Kansas) without mention of SMF. Only after we called the manufacturer, Comfort Products (Croy- 
Routine scanning protocol for scoliosis evaluation

\begin{tabular}{|c|c|c|c|c|c|c|c|c|}
\hline Series & Sequence/ETL & TR (ms) & TE (ms) & $\mathrm{ST}(\mathrm{mm})$ & NEX & FOV (mm) & SAR & Active Coil Elements \\
\hline Sag T2 upper & TSE2d/19 & 4000 & 103 & 3 & 2 & $300 \times 300$ & 1.313 & $\mathrm{H} 3,4, \mathrm{N1}, 2, \mathrm{Sl}, 2$ \\
\hline Sag T1 upper & TSE2d/3 & 682 & 9.8 & 3 & 2 & $300 \times 300$ & 1.514 & $\mathrm{H} 3,4, \mathrm{~N} 1,2$ \\
\hline Sag STIR upper & TIR2d/11 & 5400 & 18 & 3 & 1 & $300 \times 300$ & 0.331 & $\mathrm{H} 3,4, \mathrm{~N} 1,2$ \\
\hline Cor T2 upper & TSE2d/19 & 4390 & 103 & 3 & 2 & $300 \times 300$ & 1.514 & $\mathrm{H} 3,4, \mathrm{~N} 1,2$ \\
\hline Sag T2 middle & TSE2d/19 & 5310 & 103 & 4 & 2 & $300 \times 300$ & 2.634 & $\mathrm{~S} 1,2,3$ \\
\hline Sag T1 middle & TSE2d/3 & 429 & 9.8 & 4 & 2 & $300 \times 300$ & 3.67 & $\mathrm{S1}, 2,3$ \\
\hline Sag STIR middle & TIR2d/11 & 6831 & 18 & 4 & 1 & $320 \times 320$ & 0.847 & $\mathrm{S1}, 2,3$ \\
\hline Cor T2 middle & TSE2d/19 & 4620 & 103 & 4 & 2 & $320 \times 320$ & 3.436 & $\mathrm{S1}, 2,3$ \\
\hline Sag T2 lower & TSE2d/19 & 4390 & 103 & 4 & 2 & $300 \times 300$ & 2.921 & $\mathrm{~S} 2,3,4,5$ \\
\hline Sag Tl lower & TSE2d/3 & 815 & 9.8 & 4 & 2 & $300 \times 300$ & 3.425 & $\mathrm{~S} 2,3,4,5$ \\
\hline Sag STIR lower & TIR2d/9 & 5800 & 27 & 4 & 1 & $300 \times 300$ & 0.957 & $\mathrm{~S} 2,3,4,5$ \\
\hline Cor T2 lower & TSE2d/19 & 4620 & 103 & 4 & 2 & $300 \times 300$ & 2.804 & $\mathrm{~S} 2,3,4,5$ \\
\hline Axial T2 upper & TSE2d/17 & 7700 & 103 & 5 & 1 & $230 \times 230$ & 0.991 & $\mathrm{H} 4, \mathrm{~N} 2, \mathrm{~S} 1$ \\
\hline Axial T2 middle & TSE2d/17 & 7700 & 103 & 5 & 1 & $230 \times 230$ & 2.582 & $\mathrm{S1}, 2,3$ \\
\hline Axial T2 lower & TSE2d/17 & 7700 & 103 & 5 & 1 & $230 \times 230$ & 2.693 & S3,4 \\
\hline
\end{tabular}

Note:-ETL indicates echo-train length; ST, section thickness; Sag, sagittal; Cor, coronal; STIR, short tau inversion recovery; TSE2d, turbo spin-echo 2d; TIR2d, turbo inversion recovery $2 \mathrm{~d}$; coil elements: $\mathrm{H}$, head; $\mathrm{N}$, neck; $\mathrm{S}$, spine.

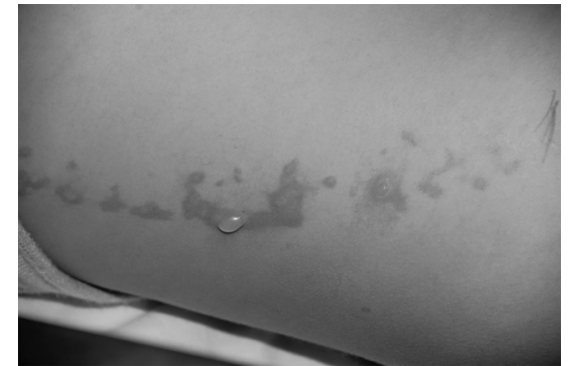

FIG 1. A linear erythematous blistering eruption is noted on the patient's right flank minutes after completion of the MR imaging of her brain and spine.

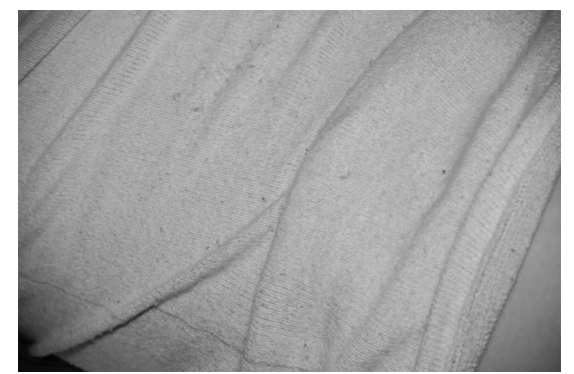

FIG 2. The patient wore a Boston Silver $T$ shirt beneath a conventional cotton tee shirt during her imaging study. It was not until after the scan that it was realized that the undershirt, recommended for wear beneath her back brace, contained antimicrobial silver microfibers.

don, Pennsylvania), was it determined that the shirt fabric was impregnated with SMF. On careful review of the shirt, it was noted that the midaxillary burn corresponded with the single shirt seam.

We hypothesize that during the study, electromagnetic eddy currents generated either within the shirt fibers concentrated at the fabric seam or between the shirt fabric seam and the patient's moist midaxillary skin resulted in significant heating and seconddegree burns.

\section{DISCUSSION}

Thermal burns are among the most commonly reported safety hazards for MR imaging patients. A recent Joint Commission
Sentinel Event Alert, citing the Manufacture and User Facility Device Experience data base of the FDA determined that $70 \%$ of MR imaging complications were related to thermal burns. ${ }^{6}$ To the best of the authors' knowledge, this is the first reported case of MR imaging - related burns associated with clothing containing invisible SMF.

MR imaging - related burns may be due to direct radio-frequency energy deposition or, more commonly, to gradient magnetic field-induced eddy currents in electrical conductors. ${ }^{7,8}$ Such currents have the potential to generate heat sufficient to cause cutaneous burns. This risk is thought to be greatest when the conductive material forms a closed loop of large diameter. ${ }^{9}$ MR imaging safety recommendations include ensuring that potentially conductive materials (eg, wires, leads, patient limbs) do not form loops and that no low-resistance electrical conductors contact the patient at $>1$ location, to minimize the possibility of forming a conductive loop involving the patient. ${ }^{9}$

We suspect that our patient sustained cutaneous burns secondary to conductive SMF embedded in her undershirt. We retrospectively imaged the undershirt by high-resolution digital radiography (Fig 3) and demonstrated an interleaving weblike pattern of radiopaque SMF. We further demonstrated the enhanced electrical conductivity of the SMF fabric relative to control polyester and cotton fabrics by measuring the resistance by using a volt/ohm multimeter. The undershirt demonstrated low resistance (on the order of $10 \mathrm{ohms}$ ), which increased monotonically as a function of the distance separating the probes. In contrast, the resistance for both the cotton and polyester fabrics was infinite.

We hypothesize that during the MR imaging examination, electromagnetic eddy currents generated within the shirt fiber concentrated at the fabric seam and led to second degree skin burns along the sites of skin contact. Because the undershirt was longer than the overlying shirt, her right wrist was in direct contact with the seam and was also affected.

Attention to the prevention of thermal burns related to clothing must be emphasized as MR imaging systems with increasing field and gradient strengths become more common. The "ACR White Paper on MR Safety" advised that all patients undergoing MR imaging must remove all clothing that contains metallic fasteners, hooks, zippers, loose metallic components, or metallic 

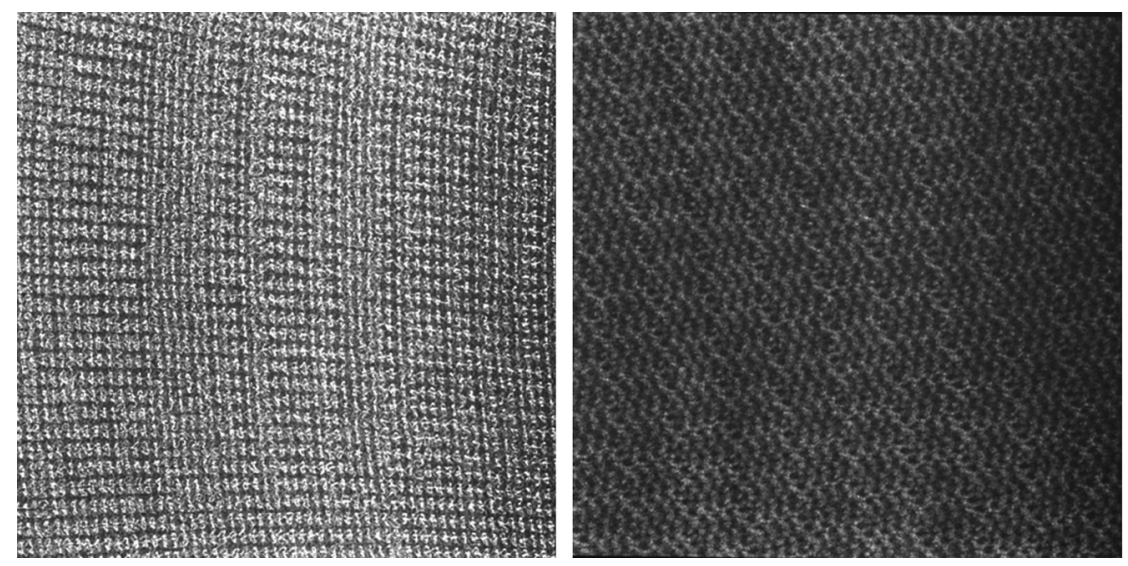

FIG 3. A digital radiograph demonstrating the metallic content of the fibers of the undershirt. Left image shows the radiopaque silver microweave fabric from the Boston Silver T. Comparison right image shows cotton fabric from a thermal undershirt.

threads. ${ }^{10}$ Furthermore, it stated that it is advisable to require that patients wear a site-supplied gown with no metal fasteners during the MR imaging procedure when feasible. ${ }^{10}$ We believe our previous policy conformed to these guidelines because we advised patients to wear a hospital-supplied gown, though we did permit patients to wear loose-fitting nonmetal-containing clothing of their own by request. Our limited survey of New England outpatient and hospital-based MR imaging facilities determined that this practice was common. We have since revised our policy and now require all patients to change into site-supplied MR imagingcompatible outer clothing and to wear nonmetallic cotton or other safe non-trade name undergarments for their examinations. Only in exceptional circumstances, because of severe physical constraints, will outpatients wearing clearly labeled $100 \%$ nonmetallic non-trade name fabrics be scanned without changing their clothing. Although fabric-content labels, regulated by the Federal Trade Commission, allow $\leq 5 \%$ impurity, ${ }^{11}$ we believe that our current policy represents a reasonable compromise between patient care and safety.

Our patient's Boston Silver T shirt showed no visible or labeling evidence to indicate that it contained SMF and was purchased to be used under external appliances (eg, a back brace) because of its perspiration wicking and antimicrobial characteristics. The manufacturer has since voluntarily modified its label to identify the silver content with the trade label X-Static (Nobel Biomaterials, Scranton, Pennsylvania) and to specifically warn against use during MR imaging examinations. The shirt still contains no attached content label.

Alarmingly, silver-impregnated textiles, marketed for antibacterial and odor-fighting properties, have seen recent expanded use $^{12-14}$ and X-Static, made with $99.9 \%$ pure silver bonded to textile fibers, ${ }^{15}$ is now incorporated in products ranging from athletic apparel (eg, golf wear, running apparel, and sports bras) to socks, orthotics, and apparel to be worn under orthoses. ${ }^{16,17}$ These products are sold under various brands including Reebok (Canton, Massachusetts), Adidas (Beaverton, Oregon), and New Balance (Boston, Massachusetts). ${ }^{17}$ Similarly, other metals with antimicrobial properties, such as copper, are being incorporated into fabrics for socks, pants, shirts, briefs, and bras ${ }^{17}$ under the trade name of Cupron and distributed by companies such as Aetrex (Teaneck, New Jersey) and Pacific Brands (Melbourne, Australia $)^{18}$ The common practice of not identifying the metallic components of multipurpose fabrics results in significant potential risk in the MR imaging environment.

Risk of burn is enhanced in the sedated patient who is unable to identify or vocalize early signs of heating. Therefore, even greater scrutiny must be used with these patients.

Use of metal detectors for screening patients before entering the scanner room is controversial. The ACR recommends using ferromagnetic detectors to primarily mitigate "missile effect" risk. This device would have been unlikely to detect SMF. We routinely use metal-detector wands to supplement patient screening. This process did not prospectively identify the metallic content of the undershirt. Retrospectively, only 1 in 4 of our wands (White's Matrix 100; New Concept Metal Detectors, Lafayette, Indiana) detected the silver content. We, therefore, cannot recommend the routine use of metal detectors to ensure clothing safety.

In summary, we demonstrate how, despite careful prescreening, invisible metallic microfiber in garments can fail to be detected before entering the MR imaging environment and potentially lead to patient thermal burns. To avoid this hazard, all patients should change into site-supplied MR imagingcompatible outer clothing and wear nonmetallic cotton or other safe non-trade name undergarments for their examinations. When exception is necessary, only patients wearing clearly labeled nonmetallic, non-trade name fabrics should be scanned without changing. We believe that those patients who are sedated or otherwise have diminished responsiveness are at greatest risk for thermal burns and, therefore, require the greatest scrutiny.

\section{REFERENCES}

1. Chaljub G, Kramer LA, Johnson RF 3rd, et al. Projectile cylinder accidents resulting from the presence of ferromagnetic nitrous oxide tanks in the MR suite. AJR Am J Roentgenol 2001;177:27-30

2. Chen DW. Boy, 6, dies of skull injury during M.R.I. New York Times. July 31, 2001:B1, B5

3. Jacob ZC, Tito MF, Dagum AB. MR imaging-related electrical thermal injury complicated by acute carpal tunnel and compartment syndrome: case report. Radiology 2010;254:846-50

4. Ratnapalan S, Greenberg M, Armstrong D. Tattoos and MRI. AJR Am J Roentgenol 2004; 183:541

5. Wagle WA, Smith M. Tattoo-induced skin burn during MR imaging. AJR Am J Roentgenol 2000;174:1795

6. The Joint Commission. Preventing accidents and injuries in the MRI suite. February 14, 2008. www.jointcommission.org/

AJNR Am J Neuroradiol 34:E47-E50 May 2013 www.ajnr.org 
sentinelevents/sentineleventalert/sea_38.htm. Accessed August 11, 2010

7. Levine GN, Gomes AS, Arai AE, et al. Safety of magnetic resonance imaging in patients with cardiovascular devices: An American Heart Association scientific statement from the Committee on Diagnostic and Interventional Cardiac Catheterization, Council on Clinical Cardiology, and the Council on Cardiovascular Radiology and Intervention: endorsed by the American College of Cardiology Foundation, the North American Society for Cardiac Imaging, and the Society for Cardiovascular Magnetic Resonance. Circulation 2007;116:2878-91. Epub 2007 Nov 19

8. Dempsey MF, Condon B. Thermal injuries associated with MRI. Clin Radiol 2001;56:457-65

9. Dempsey MF, Condon B, Hadley DM. MRI safety review. Semin Ultrasound CT MR 2002;23:392-401

10. Kanal E, Borgstede JP, Barkovich AJ. American College of Radiology White Paper on MR Safety. AJR Am J Roentgenol 2002;178:1335-47

11. Bureau of Consumer Protection. Business Bureau. Threading your way through the labeling requirements under the Textile and Wool
Acts. May 2005. http://business.ftc.gov/documents/bus21-threadingyour-way-through-labeling-requirements-under-textile-and-woolacts. Accessed February 28, 2011

12. Höfer D. Antimicrobial textiles, skin-borne flora and odour. Curr Probl Dermatol 2006;33:67-77

13. Gromer C. Smart threads. Popular Mechanics 2004;181:78-81

14. Gauger A, Mempel M, Schekatz A, et al. Silver-coated textiles reduce Staphylococcus aureus colonization in patients with atopic eczema. Dermatology 2003;207:15-21

15. X-static. The Silver Fiber. Noble Biomaterials Europe srl. http://www.Xstaticfiber.com/index 3.htm. Accessed February 27, 2011

16. Knit-Rite Inc. Knit-Rite products featuring X-STATIC. The Silver Fiber. http://www.knitrite.com/x-static-products.html. Accessed February 27, 2011

17. Noble Biomaterials. http://www.noblebiomaterials.com/page.asp? itemid $=39$. Accessed February 12, 2011

18. cupron. http://www.cupron.com/Cupron-Product-Antimicrobial. Accessed February 27, 2011 